\title{
Upcoming Events in Pediatric Cardiology
}

February 17-22, 2013

Cape Town, South Africa

World Congress Pediatric Cardiology \& Cardiac Surgery http://wcpccs2013.co.za/congress/overview

March 9-11, 2013

San Francisco, California, USA

American College of Cardiology, Annual Scientific Session http://www.acc.org

May 4-8, 2013

Minneapolis, Minnesota, USA

American Association of Thoracic Surgery, Annual Meeting

http://www.aats.org

October 26, 29, 2013

Orlando, Florida, USA

American Academy of pediatrics National Conference http://www.aapexperience.org/
October 26-31, 2013

Chicago, Illinois, USA

Annual Scientific Assembly, American College of Chest Physicians

http://www.chestnet.org/CHEST

November 16-20, 2013

Dallas, Texas, USA

Scientific Session, American Heart Association http://www.heart.org/HEARTORG/

January 25-29, 2014

Boston, Massachusetts, USA

Annual Meeting of the Society of Thoracic Surgery

http://www.sts.org

June 8-11, 2014

Chicago, Illinois, USA

Pediatric Interventional Cardiology Symposium-AICS http://www.picsymposium.com 